\title{
Electron Energy Loss Spectroscopy of Surface Plasmon Resonances on Aberrant Gold Nanostructures
}

\author{
Gregory T. Forcherio, ${ }^{\mathrm{a}, \dagger}$ Drew DeJarnette, ${ }^{\mathrm{b}, \dagger}$ Mourad Benamara, ${ }^{\mathrm{c}}$ and D. Keith Roper ${ }^{\mathrm{a}, \mathrm{d}, *}$ \\ ${ }^{a}$ MicroElectronics-Photonics Program, University of Arkansas, Fayetteville, AR 72701, USA \\ ${ }^{\mathrm{b}}$ Department of Mechanical Engineering, University of Tulsa, Tulsa, OK 74104, USA \\ ${ }^{\mathrm{c}}$ Institute for Nanoscience and Engineering, University of Arkansas, Fayetteville, AR 72701, USA \\ ${ }^{\mathrm{d}}$ Ralph E. Martin Department of Chemical Engineering, University of Arkansas, Fayetteville, AR \\ 72701, USA \\ $\dagger$ 'equally contributing authors \\ *Corresponding author. E-mail: dkroper@uark.edu, phone: (479) 575 - 6691.
}

\section{SUPPORTING INFORMATION}

This supporting document provides an illustrative, in-depth description of interpreting EELS maps for mode identification and experimental EELS data for an aberrant nanoellipse. EELS maps were generated with DDEELS v2.0; eDDA does not readily output EELS maps.

\section{S.1 Distinguishing Bright, Dark, and Hybrid Modes}

EELS can excite both "bright" and "dark" plasmon resonant modes. Bright and dark modes are uniquely characterized by the presence, or lack of a net dipole moment, $\sum \vec{p}$. "Bright" modes are resonances which exhibit a finite net dipole moment $\left(\sum \vec{p} \neq 0\right)$. "Dark" modes are resonances which exhibit a net zero dipole moment $\left(\sum \vec{p}=0\right)$. A dipole moment $\vec{p}$ is defined as the separation of charge densities $+Q$ and $-Q$ over a length $x$, such that $\vec{p}=Q \vec{x}$, where $\hat{x}$ points from negative to positive charge.

It can be helpful to view EELS maps within the context of photonic excitation. EELS results may be transposed to polarized photonic excitation by examining a linear section of the EELS map which contains (a) the structure centroid and (b) impact point, and attributing appropriate charge densities in energy loss hotspots. The charge density $Q=e N$ was given to the impact point to represent positive charge buildup around the incident electron beam, where $N$ is the total displaced carrier population. Energy loss hotspots within a given linear section were given charge densities $Q^{\prime}=-e N^{\prime}$, where $e$ is the charge of an electron and $N^{\prime}$ is the population $\left(N^{\prime} \leq N\right)$. Remaining energy loss hotspots of lower intensity were given charge densities $Q^{\prime \prime}=-e N^{\prime \prime}$, where $N^{\prime \prime}<N^{\prime}$. For simplicity, each area was labeled with a "-" or "+" sign to represent negative and positive charge, respectively, only within the considered linear section as examples. Attribution of positive/negative charge permits dipole moment identification. This method is helpful in identifying bright-dark "hybrid" resonances which exhibit a net dipole moment, but preclude 
photon excitation. "Hybrid" modes have a net dipole moment $\left(\sum \vec{p} \neq 0\right)$ arising from a sum of individual dipole moments with opposing directions arising at different locations on the structure. Subwavelength dimensions preclude simultaneous formation of each individual dipole to support the net dipole moment, thus precluding efficient coupling with light.

\section{S.2 Nanodisk EELS Maps}

Interpretation of mode-types from EELS maps requires coordinated consideration of (1) loss states supported at a given impact point (via EELS spectra) and (2) spatially adjacent point which support the same loss state (via EELS maps). It is important to recall EELS maps represent energy loss topography as opposed to charge polarization. Therefore EELS map interpretation requires attribution of corresponding charge density within a lab-frame to energy losses (relative to the impact point).

For example, a $2.0 \mathrm{eV}$ low-energy resonance is supported by edge excitation with energy losses distributed around the edge of the disc, shown in Fig. S1(a). Positive charge $(+N e)$ would build up around the impact point in a lab-frame, which was assumed as the red impact point in Fig. 1(a) inset. Electrons of $2.0 \mathrm{eV}$ energy dissipated away from this impact point are lost into the disc along the edges. In an analogy to polarized light excitation, a cross-sectional area (dotted lines) was taken along the $\mathrm{x}$-axis to illustrate the resultant dipole moments. A single dipole moment was observed between adjacent edges of the disc (see arrow), thus confirming "bright" behavior because $\sum \vec{p} \neq 0$. Furthermore, the carrier population would be conserved between positive and negative charge densities from light excitation.

The $2.4 \mathrm{eV}$ high-energy resonance supported by excitation at the disc center [mapped in Fig. S1(b)] is a "dark" mode because of dipole moment vector cancelation $\left(\sum \vec{p}=0\right)$. Positive charge will build up upon electron incidence on the disk center $(+N e)$ and negative charge will be lost uniformly to the edges $\left(-N^{\prime} e\right)$. Dipole cancellation precluded net dipole formation, independent of the cross section examined. Extensively, subwavelength dimensions of the disc preclude a polarized photon from displacing negative charge in opposing directions from the positively charged disc center.

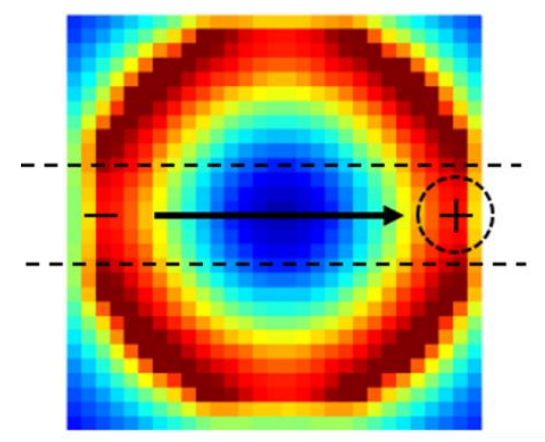

(a) $2.03 \mathrm{eV}$

bright bonding

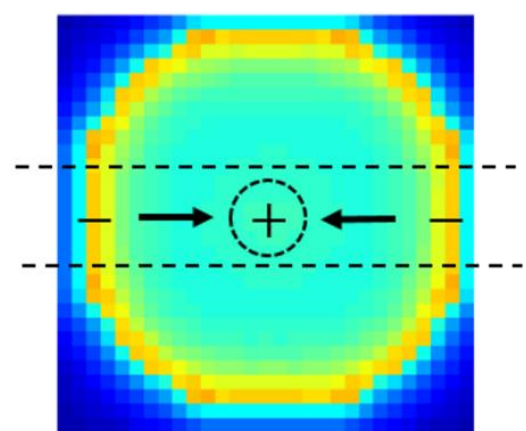

(b) $2.45 \mathrm{eV}$ dark

Figure S1. Computational EELS maps at (b) $2.03 \mathrm{eV}$ and (c) $2.45 \mathrm{eV}$ electron excitation. Resultant dipole moments (arrows) and charge distributions for $2.03 \mathrm{eV}$ and $2.45 \mathrm{eV}$ are sketched based on edge (green) and center (blue) excitation [see Fig. 1(a) inset], respectively. 


\section{S.3 Nanoring EELS Maps}

Fig. 1(c) EELS spectra illustrates bright bonding at $1.41 \mathrm{eV}$ and anti-bonding at $2.36 \mathrm{eV}$, as well as a $2.11 \mathrm{eV}$ dark mode on a nanoring. Bright bonding behavior is illustrated in Fig. S2(a) by $\sum \vec{p} \neq 0$, as found in other nanoring reports. Fig. S2(b) shows dipole cancellation $\left(\sum \vec{p}=0\right)$ within each nanoring wall, resulting in a dark mode. Electron beam incidence at the center of the ring gave equal losses near the impact point, resulting in negative charge on the inner/outer walls. Bright anti-bonding is illustrated in Fig. S2(c), where loss was concentrated the inner/outer walls. Electron beam incidence at the outer edge resulted in negative charge accumulation on the adjacent inner wall. However, the locality of EELS polarization remains obscure; the opposite ring wall could form accompanying dipole moment (as depicted) or could remain charge neutral. EELS on annular nanostructures with multiple interfaces (assuming equal in structure) orthogonal to the excitation field may require careful consideration of only isolated regions.

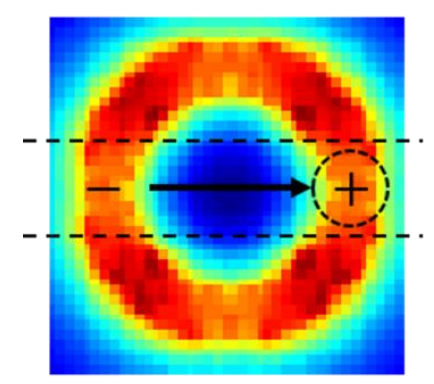

(a) $1.41 \mathrm{eV}$ bright bonding

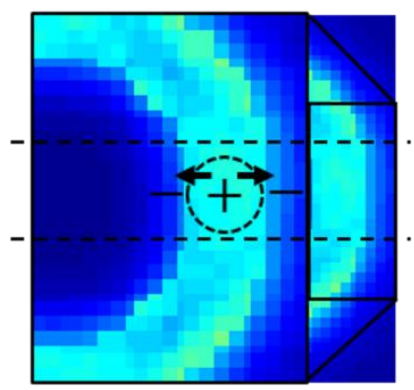

(b) $2.11 \mathrm{eV}$ dark

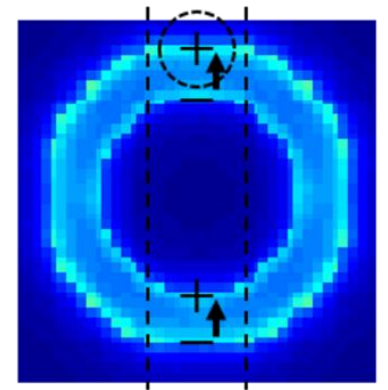

(c) $2.36 \mathrm{eV}$ bright anti-bonding

Figure S2. Computational EELS maps at (a) $1.41 \mathrm{eV}$, (b) $2.11 \mathrm{eV}$ (zoomed in), and (c) $2.36 \mathrm{eV}$ corresponding to spectra in Fig. 1(c). Resultant dipole moments (arrows) and charge distributions for $1.41 \mathrm{eV}$ and $2.11 \mathrm{eV}$, and 2.36 $\mathrm{eV}$ are sketched based on center (purple) and edge (red) excitation [see Fig. 1(c) inset], respectively.

\section{S.4 Experimental EELS for Aberrant Nanoellipse}

The plasmonic mode structure for an EBL-fabricated, aberrant Au nanoellipse with 1.5x aspect ratio, common with the eDDA simulation in Fig. 1(b), was measured using EELS. Figure S3 shows EELS spectra measured at the center, mid-point of the major axis, and edge-point of the minor axis. Using results from the in vacuo homogeneous ellipse in Fig. 1(b) as a guide, resonances measured at $1.10 \mathrm{eV}$ and 1.80 corresponded to bright modes; $1.95 \mathrm{eV}$ and $2.15 \mathrm{eV}$ corresponded to dark modes. Resonance red-shifts accrued from the $2 \mathrm{~nm} \mathrm{Cr}$ adhesion layer and $\mathrm{SiN}$ membrane were anticipated. Not-withstanding the screening-induced red-shifts, appearance of primary features in the EELS spectra were consistent with those simulated using eDDA. Impact at the mid-point of the major axis (purple) resulted in two overlapping modes appearing at 1.10 $\mathrm{eV}$ (bright) and approximately $1.95 \mathrm{eV}$ (dark); energies of the modes were sufficiently separated that the metrological $0.50 \mathrm{eV}$ energy resolution did not limit their ability to be distinguished 
empirically, based on insight from the simulation. Impact at the minor edge (green) resulted in a single mode at $1.80 \mathrm{eV}$ (bright). A dark mode (high-energy shoulder in Fig. 1(b)) would be anticipated to exist at ca. $1.95 \mathrm{eV}$ (based on results from the major mid-point), but was within the $0.50 \mathrm{eV}$ resolution threshold from the $1.80 \mathrm{eV}$ resonance, and consequently unresolvable. Impact at the center (blue) resulted in one mode at approximately $2.15 \mathrm{eV}$ (dark). Measured eigenstates were within $20 \%$ of their in vacuo counterparts, with the exception of the $1.10 \mathrm{eV}$ bright resonance whose energy was $34 \%$ lower than the simulated $1.67 \mathrm{eV}$ value. The increased error was ostensibly attributable to the mass distortion near the impact point along the major axis (see inset HAADFSTEM image), which effectively increased the major radii.

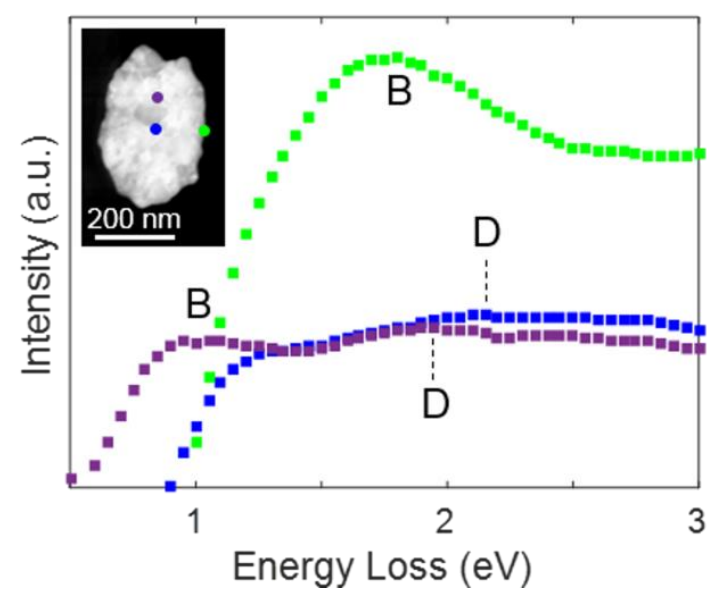

Figure S3. Measured EELS spectra for an aberrant, 1.5x aspect ratio Au nanoellipse (c.f., Fig. 1(b)) taken at the center (blue), major axis mid-point (purple), and minor axis edge (green). 